\title{
PROOF OF A THEOREM OF BURKE AND HODEL ON THE CARDINALITY OF TOPOLOGICAL SPACES ${ }^{1}$
}

\author{
ROBERT L. BLAIR
}

\begin{abstract}
Techniques of Pol are used to give a direct proof of Burke and Hodel's inequality $|X|<2^{\Delta(X) \cdot p s w(X)}$, where $\Delta(X)$ is the discreteness character of the $T_{1}$ space $X$ and $\operatorname{psw}(X)$ is the point separating weight of $X$.
\end{abstract}

A cover $\mathcal{G}$ of a topological space $X$ is separating if for each $x, y \in X$ with $x \neq y$ there is $G \in \mathcal{G}$ with $x \in G$ and $y \notin G$. For a $T_{1}$ space $X$ the point separating weight $\operatorname{psw}(X)$ of $X$ is $\omega \cdot \mathfrak{n}$, where $\mathfrak{n}$ is the smallest cardinal such that $X$ has a separating open cover $\mathcal{G}$ with ord $(x, \mathcal{G}) \leqslant \mathfrak{n}$ for all $x \in X$, and the discreteness character $\Delta(X)$ of $X$ is $\omega \cdot \mathfrak{n}$, where $\mathfrak{n}=\sup \{|D|: D$ is a closed discrete subset of $X\}$ (see [BH]). In [BH, 4.4] Burke and Hodel use a version, due to Burke [BH, 4.1], of the ErdösRado $\Delta$-system lemma to prove the theorem below. Here we give a direct proof suggested by techniques of Pol $\left[\mathbf{P}_{\mathbf{1}}\right]$. (These in turn derive from ideas of Ponomarev $\left[\mathbf{P}_{2}\right]$ and Sapirovskiı [S]; see also [E, 3.12.10] and [BH, 4.6].)

TheOREM (Burke AND Hodel). If $X$ is $T_{1}$, then $|X| \leqslant 2^{\Delta(X) \cdot p s w(X)}$.

Proof. Let $\mathfrak{m}=\Delta(X) \cdot \operatorname{psw}(X)$, let $\mathcal{G}$ be a separating open cover of $X$ with $\operatorname{ord}(x, \mathcal{G}) \leqslant \mathfrak{m}$ for all $x \in X$, and for each $x \in X$ let $\mathcal{G}_{x}=\{G \in \mathcal{G}: x \in G\}$. Construct a sequence $\left(Y_{\xi}\right)_{\xi<\mathfrak{m}^{+}}$such that:

(1) For each $\xi<\mathfrak{m}^{+}, Y_{\xi} \subset X$ and $\left|Y_{\xi}\right| \leqslant 2^{\mathfrak{m}}$.

(2) If $\eta \leqslant \xi<\mathfrak{m}^{+}$, then $Y_{\eta} \subset Y_{\xi}$.

(3) If $0<\xi<\mathfrak{m}^{+}$and $\mathscr{U} \subset \cup\left\{\mathcal{G}_{x}: x \in \cup_{\alpha<\xi} Y_{\alpha}\right\}$ with $|\mathscr{Q}| \leqslant \mathfrak{m}$ and $X-$ $\cup$ थ $\neq \varnothing$, then $Y_{\xi}-\cup$ थ $\neq \varnothing$.

(Set $Y_{0}=\varnothing$. If $0<\xi<\mathrm{m}^{+}$and $Y_{\alpha}$ is already defined for all $\alpha<\xi$, let $E$ be the set obtained by choosing a point from each nonempty member of $\{X-\cup$ U: $\left.\mathscr{U} \subset \cup\left\{\mathcal{G}_{x}: x \in \cup_{\alpha<\xi} Y_{\alpha}\right\},|\mathscr{Q}| \leqslant \mathfrak{m}\right\}$ and set $Y_{\xi}=E \cup\left(\cup_{\alpha<\xi} Y_{\alpha}\right)$.)

Let $Y=\bigcup_{\xi<\mathfrak{m}^{+}} Y_{\xi}$. It suffices to show that $Y=X$. Suppose $p \in X-Y$. For each $x \in Z=X-\{p\}$ there is $G_{x} \in \mathcal{G}_{x}$ with $p \notin G_{x}$. Let $\mathcal{V}=\left\{G_{x}: x \in Z\right\}$ and, by Zorn's lemma, choose $D \subset Z$ such that $D \cap \operatorname{st}(x, \mathcal{V})=\{x\}$ for all $x \in D$ and $Z \subset \cup_{x \in D} \operatorname{st}(x, \mathscr{V})$. Let $\mathscr{Q}=\{U \in \mathcal{V}: U \cap D \neq \varnothing, U \cap Y \neq \varnothing\}$. Note that

Received by the editors March 26, 1979.

AMS (MOS) subject classifications (1970). Primary 54A25.

Key words and phrases. Cardinality of a topological space, separating open cover, point separating weight, discreteness character.

${ }^{1}$ This research was supported in part by Ohio University Research Committee Grant No. 535. 
$D$ is closed discrete in $Z=\cup\left\{X-G: G \in \mathcal{G}_{p}\right\}$, so $|D|<m$ and hence $|\mathscr{Q}|<$ m. Then there is $\beta<\mathfrak{m}^{+}$with $U \cap Y_{\beta} \neq \varnothing$ for all $U \in \mathcal{U}$. Clearly $\mathscr{U} \subset \cup\left\{\mathcal{G}_{x}\right.$ : $\left.x \in \cup_{\alpha<\beta+1} Y_{\alpha}\right\}$ and $p \in X-\cup \mathcal{U}$, but $Y_{\beta+1} \subset Y \subset \cup_{x \in D} \operatorname{st}(x, \mathscr{V})$ and hence $Y_{\beta+1}-\cup \mathscr{U}=\varnothing$, a contradiction.

\section{REFERENCES}

[BH] D. K. Burke and R. E. Hodel, The number of compact subsets of a topological space, Proc. Amer. Math. Soc. 58 (1976), 363-368.

[E] R. Engelking, General topology, PWN, Warsaw, 1975; English Transl., PWN, Warsaw, 1977.

[P, R. Pol, Short proof of two theorems on cardinality of topological spaces, Bull. Acad. Polon. Sci. Sér. Sci. Math. Astronom. Phys. 22 (1974), 1245-1249.

$\left[\mathbf{P}_{2}\right]$ V. I. Ponomarev, On the cardinality of bicompacta which satisfy the first axiom of countability, Dokl. Akad. Nauk SSSR 196 (1971), 296-298 = Soviet Math. Dokl. 12 (1971), 121-124.

[S] B. Šapirovskii, On discrete subspaces of topological spaces: weight, tightness and Suslin number, Dokl. Akad. Nauk SSSR 202 (1972), 779-782 = Soviet Math. Dokl. 13 (1972), 215-219.

Department of Mathematics, Oho University, Athens, OHo 45701 